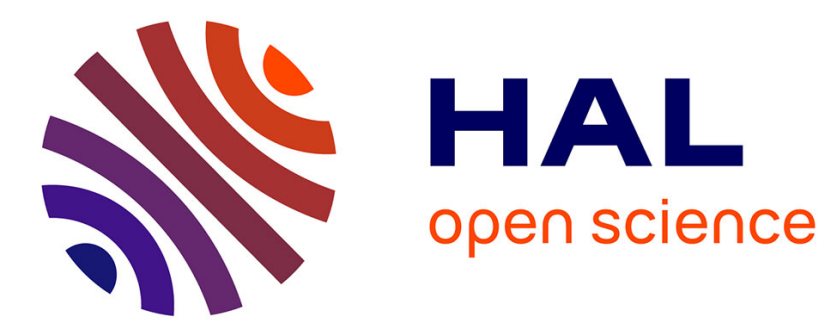

\title{
Simulation of laser-driven cratering experiments on aluminum
}

\author{
Bertrand Aubert, David Hebert, Jean-Luc Rullier, Emilien Lescoute, Laurent \\ Videau, Laurent Berthe
}

\section{> To cite this version:}

Bertrand Aubert, David Hebert, Jean-Luc Rullier, Emilien Lescoute, Laurent Videau, et al.. Simulation of laser-driven cratering experiments on aluminum. Journal of Laser Applications, 2019, 31 (4), pp.1-10. 10.2351/1.5086736 . hal-02983554

\section{HAL Id: hal-02983554 https://hal.science/hal-02983554}

Submitted on 30 Oct 2020

HAL is a multi-disciplinary open access archive for the deposit and dissemination of scientific research documents, whether they are published or not. The documents may come from teaching and research institutions in France or abroad, or from public or private research centers.
L'archive ouverte pluridisciplinaire HAL, est destinée au dépôt et à la diffusion de documents scientifiques de niveau recherche, publiés ou non, émanant des établissements d'enseignement et de recherche français ou étrangers, des laboratoires publics ou privés. 


\title{
Simulation of laser-driven cratering experiments on aluminum
}

\author{
Bertrand Aubert, ${ }^{1, \text { a) }}$ David Hebert, ${ }^{7}$ Jean-Luc Rullier, ${ }^{1}$ Emilien Lescoute, ${ }^{2}$ Laurent Videau, ${ }^{2}$ and Laurent Berthe ${ }^{3}$
}

AFFILIATIONS

${ }^{1}$ CEA CESTA, 15 Avenue des Sablieres, CS60001, 33116 Le Barp Cedex, France

${ }^{2}$ CEA DIF, Bruyeres-le-Chatel, 91297 Arpajon Cedex, France

${ }^{3}$ Laboratoire PIMM—UPR80006 CNRS-ENSAM, 151 Boulevard de l'Hôpital, 75013 Paris, France

\begin{abstract}
After a brief description of the physical principles involved in the cratering process, the authors present a specific methodology to simulate laser-driven cratering experiments performed with a long pulse duration (100 ns) and a small focal spot diameter $(220 \mu \mathrm{m})$. This methodology can be divided into two steps. First, the 2D-axisymmetrical pressure field generated by the laser on the target is determined from laser parameters. Second, this pressure is applied on the surface of the target in a Eulerian simulation. In order to validate this methodology, the authors simulate a laser shot on a thin aluminum target whose rear surface velocity is recorded by a VISAR (Velocity Interferometer System for Any Reflector). Once validated, they use the methodology to simulate laser-driven cratering experiments on semi-infinite aluminum targets. Numerical results are compared to experimental measurements of the craters. Although slight differences are pointed out and discussed, the proposed methodology is well adapted to simulate craterization laser shots.
\end{abstract}

Key words: laser, crater, aluminum, simulation

\section{INTRODUCTION}

The number of space debris occupying Earth's orbital space is constantly increasing. Satellites and spacecrafts must be designed to resist impacts of several kilometers per second with such debris. ${ }^{1}$ Usually, launchers are used to recreate this type of impact in the laboratory, but it is difficult to reach very high impact velocities with large projectiles (typically above $10 \mathrm{~km} \mathrm{~s}^{-1}$ for a projectile of millimeter size). ${ }^{2,3}$ It has been observed since the 1970 s that laser irradiation could be used to generate similar damage. ${ }^{4-8}$

For many laser applications, the focal spot size used is large compared to the dimensions of the sample, and laser-driven shock simulations are usually performed with the monodimensional Lagrangian code. ${ }^{9,10}$ For smaller focal spot size or thicker samples, two-dimensional ${ }^{1,12}$ or three-dimensional ${ }^{13}$ simulations are required. Moreover, if the deformations induced by the laser shock are too large, Lagrangian simulations are no longer suitable and an alternative must be found, such as Eulerian simulations. If the laser deposit is sufficiently brief and ends before large deformations appear, then it is possible to use the Lagrangian mode during the deposit and then to skip to the Eulerian mode for the rest of the simulation. ${ }^{7,14}$

In the present paper, we focus on numerical simulations of laser-driven cratering experiments performed with the GCLT laser facility (Generateur de Choc Laser Transportable) on aluminum targets. During the cratering process, very large deformations are observed. Furthermore, a long pulse duration is used for this study (100 ns), so large deformations appear while the laser deposit is not yet finished. This prevents us from starting the simulation in the Lagrangian mode and continuing it in the Eulerian mode. As a consequence, we chose to use a Eulerian scheme throughout the simulation.

In Sec. II, the main physical principles involved in the craterization process are described. Similarities between projectile impacts and laser irradiations are pointed out. In Sec. III, we propose a complete methodology adapted to simulate laser-driven cratering experiments, and we present the numerical tools that have been used. Two different types of experiments have been performed during this study: the first one to validate our methodology and the second one to apply it. The two experimental setups are presented in Sec. IV. In 
Sec. V, the methodology is validated, thanks to a laser shot performed on a thin aluminum target instrumented with the VISAR (Velocity Interferometer System for Any Reflector). ${ }^{15}$ Finally, in Sec. VI, the methodology is applied to the simulation of laser-driven cratering experiments on thick aluminum targets.

\section{PHYSICAL PRINCIPLES}

Under certain conditions, a projectile impact can lead to the formation of a crater. It depends on the impact velocity, the stiffness of materials, and the relative size of the projectile compared to the target thickness. In this study, we are interested in hypervelocity impacts of ductile projectiles on semi-infinite ductile targets. This type of impact always generates craters. Temporally, the formation of these craters can be divided into three steps: ${ }^{16}$

1. Shock waves are generated and propagate into both the target and the projectile leading to the formation of a crater in the target and the deformation of the projectile.

2. Crater expands until the shock waves have been sufficiently attenuated to no longer be able to deform the target material.

3. Crater volume may decrease slightly by an elastic rebound and temperature slowly goes back to the ambient inducing recrystallization of the material.

Similarly, it is possible to generate a crater by focusing a high power laser on a semi-infinite target. ${ }^{5}$ The process of formation can be described as follows:

1. In the first moments of the interaction, laser irradiation is absorbed in the skin depth of the solid target due to the inverse bremsstrahlung mechanism.

2. Surface matter rapidly reaches its vaporization temperature leading to the formation of a plasma. Progressively, a continuous regime of ablation is established: laser energy is deposited in the plasma area where electronic density is close to the critical density and partially transported to the solid target by conduction leading to a new material evaporation which feeds the plasma.

3. During its rapid expansion, the plasma applies a pressure on the surface of the target. This pressure, called ablation pressure, generates a shock wave in the target.

4. As for a projectile impact, this shock wave generates a crater which grows until the attenuation of the wave and potentially decreases by an elastic rebound.

Thermal effects are probably the main difference between projectile impacts and laser irradiation. The plasma generated by the laser can reach very high temperature (typically $>10^{6} \mathrm{~K}$ ), whereas a projectile impact induces temperature of only a few hundreds of kelvin in the target. However, if the thickness ablated by the laser is sufficiently thin compared to the crater depth, thermal effects may not significantly affect the cratering process. In this case, it seems possible to use laser facilities to reproduce projectile impacts.

\section{METHODOLOGY AND NUMERICAL TOOLS}

In order to establish an analogy between projectile impact and laser irradiation, it is essential to know the pressure induced by the laser on the target because it is the link between the two processes.
Some empirical formulas ${ }^{5,17-19}$ allow one to evaluate the ablation pressure knowing laser parameters and target properties, but it has been shown that numerical simulations were more accurate. ${ }^{20}$ The aim of this section is to propose a methodology adapted to simulate a laser-driven craterization shot and, therefore, to determine the ablation pressure.

A global simulation of the craterization process requires a code able to manage laser/matter interaction (beam propagation, reflection, absorption, ionization, conduction, hydrodynamics of evaporation, and plasma expansion) and mechanical response of the target (hydrodynamics of shock waves propagation, elastoplasticity, and damage). However, the scales of these phenomena are very different temporally and spatially. On the one hand, the mesh size near the target surface cannot exceed $10^{-9} \mathrm{~m}$ to correctly reproduce the laser deposit so we have to use time steps of about $10^{-13} \mathrm{~s}$ with respect to the CFL condition. On the other hand, the time of the formation of a crater is of about $10^{-6} \mathrm{~s}$, so the number of cycles required for a complete simulation is very significant.

In order to reduce computational time, we decided to split the two phenomena by coupling two different codes: the ESTHER code to simulate the laser/mater interaction and the HESIONE code to simulate the shock propagation in the target and the crater formation. The two codes are linked by the ablation pressure as described below.

For each laser shot, the following parameters are known: energy delivered to the target $E$, the temporal profile of the impulsion $f(t)$, and the spatial distribution of intensity $g(r)$. The fluence at the center of the focal spot $F(r=0, t)$ can also be determined from these parameters. This fluence and material properties of the target are used as input data of an ESTHER simulation. ESTHER is a Lagrangian monodimensional code developed by the French Atomic Energy Commission (CEA). It is able to manage the laser/ matter interaction and the shock propagation from the picosecond regime to the nanosecond regime. ${ }^{20-22}$ It solves the following system of conservation equations:

$$
\begin{gathered}
\frac{\partial \rho}{\partial t}+\nabla \cdot(\rho \vec{v})=0, \\
\frac{\partial(\rho \vec{v})}{\partial t}+\nabla \cdot[\rho \vec{v} \otimes \vec{v}+p \overline{\bar{I}}]=\overrightarrow{0}, \\
\frac{\partial\left(\rho e_{\text {tot }}\right)}{\partial t}+\nabla \cdot\left(\rho e_{\text {tot }}+p \overline{\bar{I}} \vec{v}\right)=\nabla \cdot(\lambda \nabla T)+\frac{\sigma_{r}}{2}\|\vec{E}\|^{2},
\end{gathered}
$$

where $t$ is the time, $\rho$ is the density, $\vec{v}$ is the velocity, $p$ is the hydrostatic pressure, $e_{\text {tot }}$ is the total energy defined as $e_{\text {tot }}=e_{\text {int }}+\frac{1}{2} \vec{v}^{2}, \lambda$ is the thermal conductivity, $T$ is the temperature, and $\sigma_{r}$ is the real part of the complex electric conductivity $\tilde{\sigma}$. The complex electric field, denoted $\vec{E}$, is determined, thanks to the Helmholtz equation,

$$
\Delta \vec{E}+\left(\frac{2 \pi}{\lambda_{0}} \tilde{n}\right)^{2} \vec{E}=\overrightarrow{0}
$$

where $\lambda_{0}$ is the wavelength of the laser and $\tilde{n}$ is the complex index of refraction in the plasma. To close the system of equations, we use an 
equation of state which provides a thermodynamically consistent relation between $P, e_{\text {int }}, \rho$, and $T$. Note that material parameters $\tilde{\sigma}$, $\tilde{n}$, and $\lambda$ have to be known over a wide range of pressure and temperature from a solid to plasma state. The data we used for our specific case will be presented in Sec. V B.

From ESTHER simulation, we can extract the ablation pressure generated at the center of the focal spot $P_{\mathrm{ab}}(r=0, t)$. Combining this monodimensional ablation pressure law with the spatial distribution of intensity $g(r)$ measured experimentally, we build the $2 \mathrm{D}$-axisymmetrical pressure field generated by the laser $P_{\mathrm{ab}}(r, t)$.

Then, this pressure field is used as a boundary condition of an HESIONE simulation. HESIONE is a hydrodynamic code developed by the CEA which can be used in either Lagrangian or Eulerian mode to simulate the mechanical response of a material under dynamic loading in 1D, 2D, 2D-axisymmetrical, or 3D representation. It solves the following three conservation equations:

$$
\begin{gathered}
\frac{\partial \rho}{\partial t}+\nabla \cdot(\rho \vec{v})=0, \\
\frac{\partial(\rho \vec{v})}{\partial t}+\nabla \cdot[\rho \vec{v} \otimes \vec{v}-\overline{\bar{\sigma}}]=\overrightarrow{0} \\
\frac{\partial\left(\rho e_{\mathrm{tot}}\right)}{\partial t}+\nabla \cdot\left(\rho e_{\mathrm{tot}}-\overline{\bar{\sigma}} \vec{v}\right)=0,
\end{gathered}
$$

where $\overline{\bar{\sigma}}$ is the stress tensor defined by $\overline{\bar{\sigma}}=-p \overline{\bar{I}}+\overline{\bar{S}}$, with $\overline{\bar{S}}$ being the deviatoric stress tensor. An equation of state is used to close this system of equations. For isotropic materials, $\overline{\bar{S}}$ is given by the following incremental constitutive relation:

$$
\frac{d \overline{\bar{S}}}{d t}=2 G\left[\overline{\bar{D}}-\frac{1}{3} \operatorname{trace}(\overline{\bar{D}}) \overline{\bar{I}}\right]+\overline{\bar{\Omega}} \overline{\bar{S}}-\overline{\bar{S}} \overline{\bar{\Omega}},
$$

where $G$ is the shear modulus and

$$
\begin{aligned}
& \overline{\bar{D}}=\frac{1}{2}\left[\nabla \otimes \vec{v}+(\nabla \otimes \vec{v})^{t}\right], \\
& \overline{\bar{\Omega}}=\frac{1}{2}\left[\nabla \otimes \vec{v}-(\nabla \otimes \vec{v})^{t}\right] .
\end{aligned}
$$

The plasticity criterion associated with this behavior law is based on the Von Mises relation which can be written as

$$
\sqrt{\frac{3}{2} \overline{\bar{S}}: \overline{\bar{S}}} \leq Y
$$

where $Y$ is the yield strength. The expressions of $G$ and $Y$ used for our study will be presented in Sec. V B.

All the HESIONE simulations presented in this paper were performed in the Eulerian mode using the BBC numerical scheme ${ }^{23}$ and a 2D-axisymmetrical representation. In the Eulerian mode, HESIONE solves multimaterial flow equations on a Cartesian grid. In each cell, each material is represented by its volume fraction, so the concept of boundary is not clear. Consequently, we use a

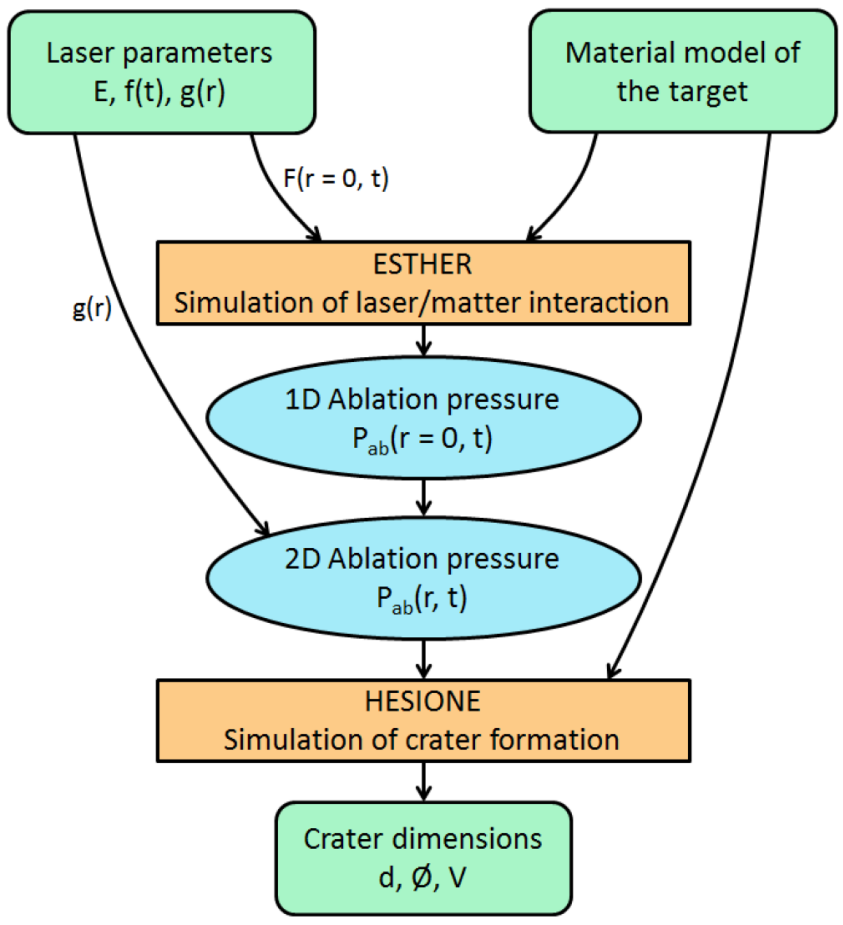

FIG. 1. Methodology proposed to simulate a laser-driven craterization shot.

fictitious domain to apply the laser-induced pressure. This technique consists of imposing the pressure within a fictitious material placed in front of the target.

Finally, craters' dimensions (depth, diameter, and volume) are extracted from the simulation. They are directly comparable with experimental measurements for the corresponding crater.

The complete methodology is summarized in Fig. 1.

\section{EXPERIMENTAL SETUP}

The laser used for our experiments is the GCLT laser facility located at CEA-DIF (France). This Nd:YAG high power laser delivers energies from 1 to $40 \mathrm{~J}$ with an adjustable pulse duration from 5 to $100 \mathrm{~ns}$ at $1057 \mathrm{~nm}$ wavelength. Several phase plates are available to shape the focal spot to the desired size. Intensities up to $10^{13} \mathrm{~W} \mathrm{~cm}^{-2}$ can be reached, so the target is positioned inside a vacuum chamber to avoid a breakdown in ambient air. The pressure in the chamber is close to 0.05 mbar.

For all the laser shots considered in this paper, the $100 \mathrm{~ns}$ impulsion was used along with a focal spot diameter of about $220 \mu \mathrm{m}$. For each laser shot, we record the temporal profile $f(t)$ of the pulse and the amount of energy $E$ delivered on the target. Once a day, we measure the spatial distribution of energy using a CCD camera placed at the target position. This spatial distribution was stable during all the experiments. Figure 2(a) shows the temporal profile for a regular shot. We note that there are some oscillations with a temporal periodicity of $\sim 5 \mathrm{~ns}$, which are due to the laser source. Figure 2(b) shows the spatial distribution of energy. Despite 
the presence of a phase plate, we observe some inhomogeneities in the focal spot. Their characteristic size is close to $35 \mu \mathrm{m}$.

Figure 2(b) can be seen as a matrix whose each element corresponds to a CCD sensor of the camera. The value measured by each captor is proportional to the energy received by this sensor. Thus, by summing all the elements of the matrix and multiplying this sum by the surface of a sensor, we obtain a quantity directly proportional to the total energy delivered by the laser. To generate a 2D-axisymmetrical profile $g(r)$ from Fig. 2(b), the following tasks are applied to the matrix:

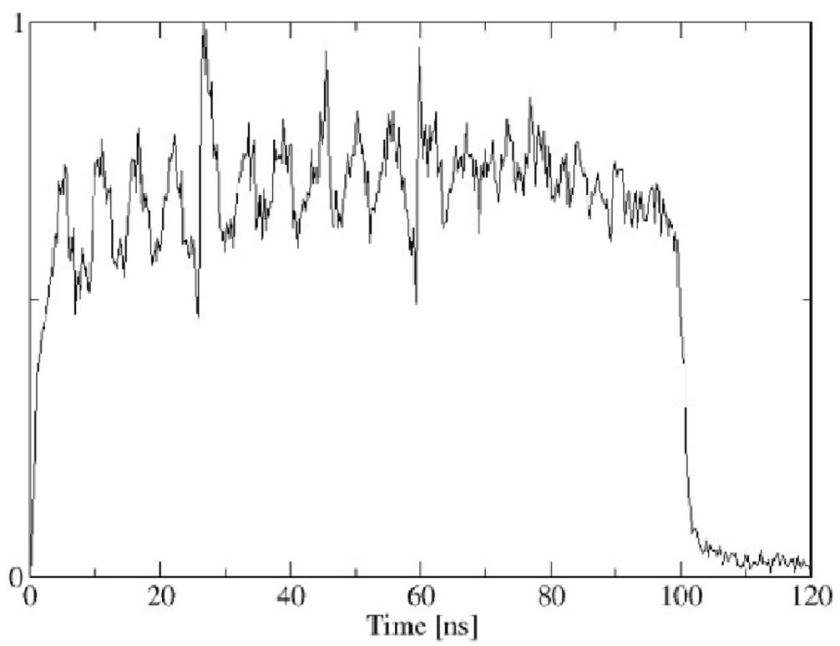

(a)

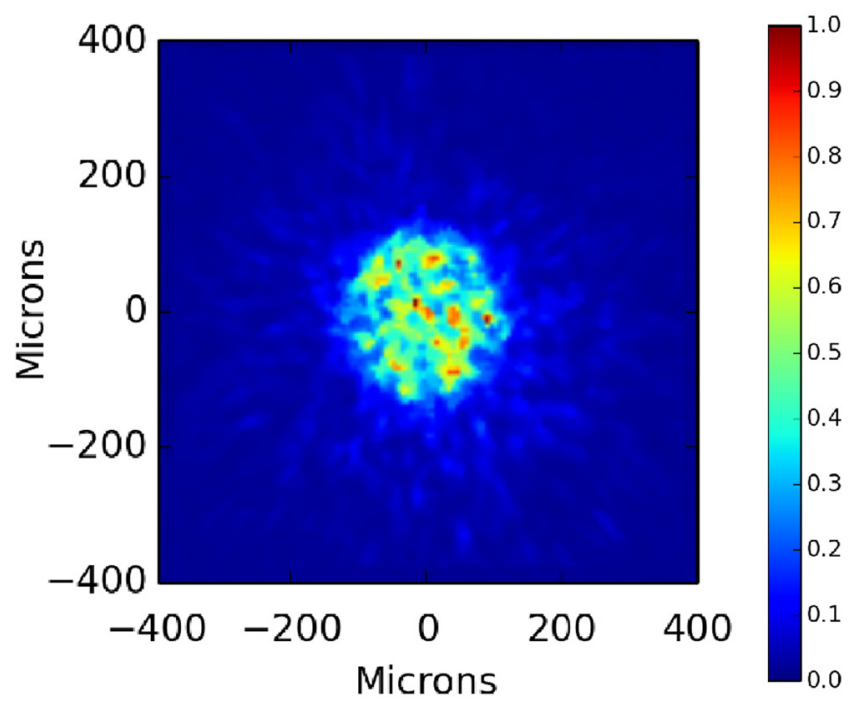

(b)

FIG. 2. Normalized temporal profile (a) and spatial distribution of energy (b) for a typical laser shot with a $250 \mu \mathrm{m}$ focal spot diameter on the GCLT facility.
1. calculation of the centroid,

2. calculation of the mean fluence $F_{\text {mean }}$ of the central plateau of the focal spot (we consider that the plateau corresponds to pixels within $70 \mu \mathrm{m}$ of the centroid),

3. simplification of the focal spot by replacing all values of the plateau by $F_{\text {mean }}$,

4. calculation of a radial profile denoted $\tilde{g}(r)$ by circular average around the centroid,

5. simplification of $\tilde{g}(r)$ for $r>300 \mu \mathrm{m}$ using an exponential law to correct measurement noise around the spot, and

6. determination of $g(r)$ proportional to $\tilde{g}(r)$ and satisfying the relation $\int_{0}^{\infty} 2 \pi r g(r) d r=E$.

The result of this procedure is shown in Fig. 3, where the dotted line indicates the extrapolation used to eliminate the measurement noise. Two points may seem questionable in this procedure. The first point is the validity of using an average fluence $F_{\text {mean }}$ on the central plateau (item 2). This approximation was necessary to smooth the inhomogeneities in the focal spot and obtain an axisymmetrical intensity profile. The second point concerns the exponential extrapolation (item 5). We will validate these choices in Sec. $V$ with experiments on thin aluminum targets.

Targets were all made of 6061-T6 aluminum. Two different thicknesses have been used: $188 \pm 2 \mu \mathrm{m}$ for validation experiments and about $3 \mathrm{~mm}$ for cratering experiments. In the first case, free surface velocities are recorded using a VISAR. ${ }^{15}$ In the second case, resulting craters are measured with an optical profilometer (Bruker Contour GT). Figure 4 is a schematic view of the two experimental setups. In order to characterize the laser loading spatially, four laser shots are performed on thin targets with different positions of the VISAR recording. The first VISAR record is located on the symmetry axis $(r=0 \mu \mathrm{m})$, and the second one near the edge of the laser profile $(r=250 \mu \mathrm{m})$. The two other records are clearly located out of the laser spot $(r=500 \mu \mathrm{m}$ and $r=750 \mu \mathrm{m})$. Good repeatability

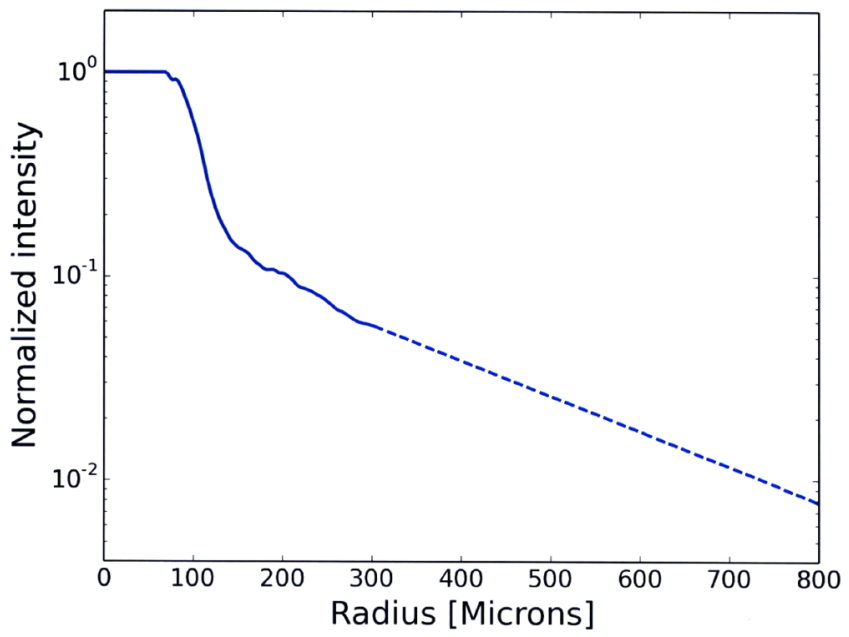

FIG. 3. Normalized 2D-axisymmetrical intensity profile $g(r)$ obtained for the focal spot presented in Fig. 2(b). 
of the laser allows us to consider that the four shots are identical and equivalent to a single shot with four different VISAR measurements, as shown in Fig. 4(a).

\section{VALIDATION OF THE METHODOLOGY}

\section{A. Experimental results}

Four laser shots have been performed with different positions of the VISAR recording as shown in Fig. 4(a). All the targets had the same thickness of $188 \pm 2 \mu \mathrm{m}$ and all the laser pulses had the same energy of $39.5 \pm 0.6 \mathrm{~J}$ with almost identical temporal profiles. Experimental measurements of free surface velocities are shown in Fig. 5.

Since the pulse duration ( $100 \mathrm{~ns})$ is longer than the transit time of the shock through the target ( $30 \mathrm{~ns})$, it is an almost steady shock configuration. When the shock reaches the free surface, a first velocity jump is observed at $30 \mathrm{~ns}$ and a release wave is generated. This wave travels back through the sample. When it reaches the front surface, it
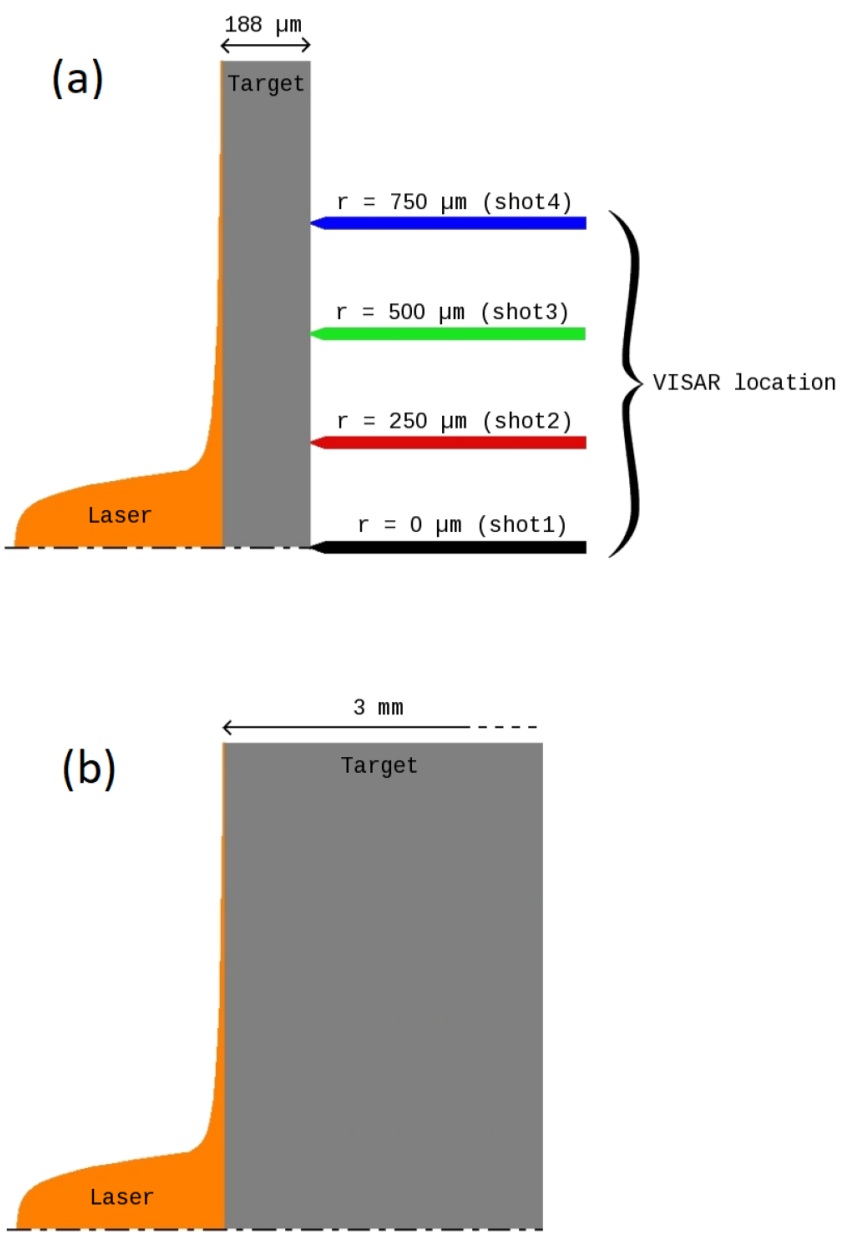

FIG. 4. Schematic of the setups used for validation experiments (a) and cratering experiments (b).

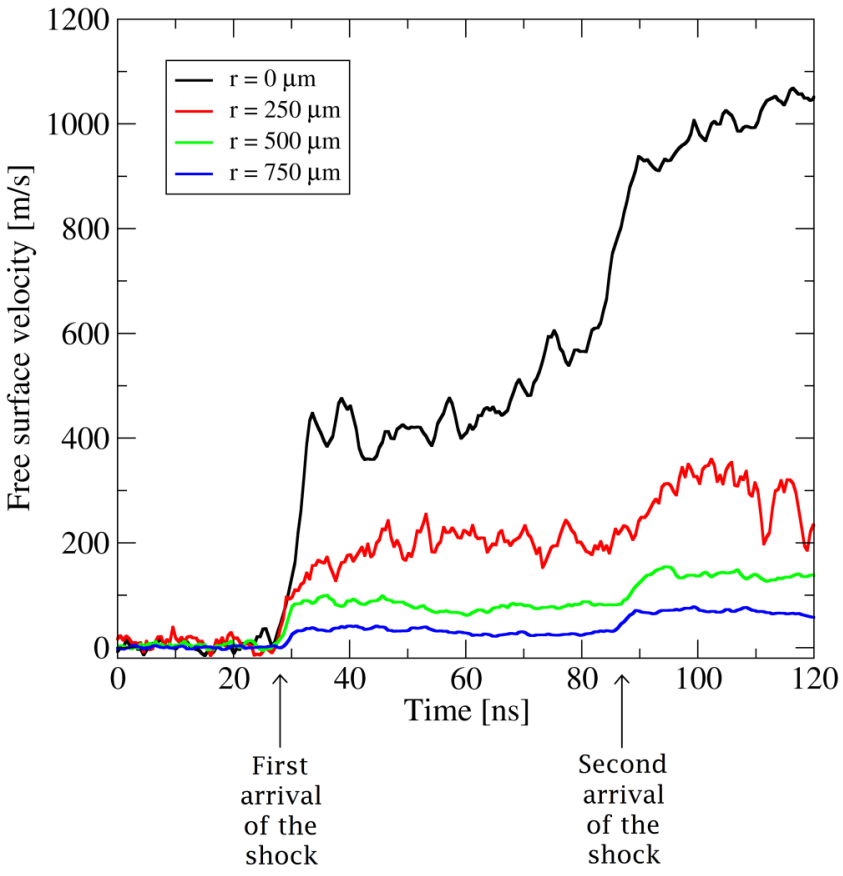

FIG. 5. Free surface velocities measured by the VISAR in four different positions for a $39.5 \mathrm{~J}$ shot with a $100 \mathrm{~ns}$ pulse duration and a $220 \mu \mathrm{m}$ focal spot diameter on a $188 \mu \mathrm{m}$ aluminum target. Time $t=0 \mathrm{~ns}$ corresponds to the beginning of laser irradiation and time $t=100 \mathrm{~ns}$ to its end.

is reflected into a reshock. The second velocity jump observed at 90 ns corresponds to the arrival of this reshock at the rear surface. Some oscillations are present in the velocity records with an approximate period of $5 \mathrm{~ns}$ and an amplitude of $\pm 10 \%$. Very similar oscillations are present in the temporal profile shown in Fig. 2(a); they are probably due to fluctuations of the laser intensity during the pulse. Furthermore, we note that the velocity is still significant $\left(\sim 50 \mathrm{~m} \mathrm{~s}^{-1}\right)$ at a radius of $750 \mu \mathrm{m}$ although it is very far from the beam edge. Moreover, the sphericity of the shock wave induces a slight difference of arrival time of the shock between the four VISAR positions.

\section{B. Numerical simulations}

In the first step, $F_{\text {mean }}$ and $f(t)$ are used as input data of an ESTHER simulation. In this simulation, hydrodynamic behavior of aluminum is described with the equation of state SESAME 3720 and the parameters $\tilde{\sigma}, \tilde{n}$, and $\lambda$ (see Sec. III) are calculated by interpolation from data in the following asymptotic regimes:

- near to the standard state, ${ }^{24,25}$

- in warm and dense plasma $\left(\rho>10^{-3} \mathrm{~g} \mathrm{~cm}^{-3}\right),{ }^{26}$ and

- in warm and rarefied plasma $\left(\rho<10^{-3} \mathrm{~g} \mathrm{~cm}^{-3}\right)$.

As output data, we obtain a $1 \mathrm{D}$ ablation pressure law $P_{\text {ESTHER, which }}$ corresponds to the ablation pressure $P_{\mathrm{ab}}$ at the center of the focal spot,

$$
P_{\text {ESTHER }}(t)=P_{\mathrm{ab}}(r=0, t) .
$$


Assuming that the radial profile $g(r)$ (Fig. 3) does not depend on the intensity level, we can write

$$
I(r, t)=I(r=0, t) g(r)
$$

Some simulations have been performed with the ESTHER code at various laser intensities to establish a relation between the maximum ablation pressure and the laser intensity (Fig. 6). We observed that $P_{\mathrm{ab}} \propto I^{0.7}$. The 0.7 exponent is consistent with the literature which propose exponents between 0.66 and $0.8^{5,17-19}$

Consequently, we obtain a $2 \mathrm{D}$-axisymmetrical ablation pressure field,

$$
P_{\mathrm{ab}}(r, t)=P_{\mathrm{ESTHER}}(t) g(r)^{0.7} .
$$

In our case, this pressure field is presented in Fig. 7. Spatially, the pressure decreases slowly following the radial profile $g(r)$ at a power of 0.7. Temporally, the pressure is close to a top-hat of about $100 \mathrm{~ns}$ followed by a slow decrease. The oscillations observed are the consequence of the oscillations present in the temporal profile of intensity because they have the same period.

This pressure field can then be applied as a boundary condition in the HESIONE hydrocode using a fictitious material placed in front of the target. In this material, the pressure always satisfies the following relation:

$$
P(r, t)=P_{\mathrm{ab}}(r, t) .
$$

By pressing the surface of the target, the fictitious material reproduces the behavior of the plasma.

In the HESIONE simulation, the behavior of 6061-T6 aluminum is described with the equation of state SESAME 3720 and the SCG model. ${ }^{28}$ With this model, rheological parameters are affected by strain hardening, pressure hardening, and thermal softening effects. Concretely, the yield strength $Y$ and the shear modulus $G$ depend on density $\rho$, pressure $P$, temperature $T$, and plastic strain $\epsilon$. If the temperature is higher than the melting temperature of the material, then $G$ and $Y$ are set to zero, else they are governed by

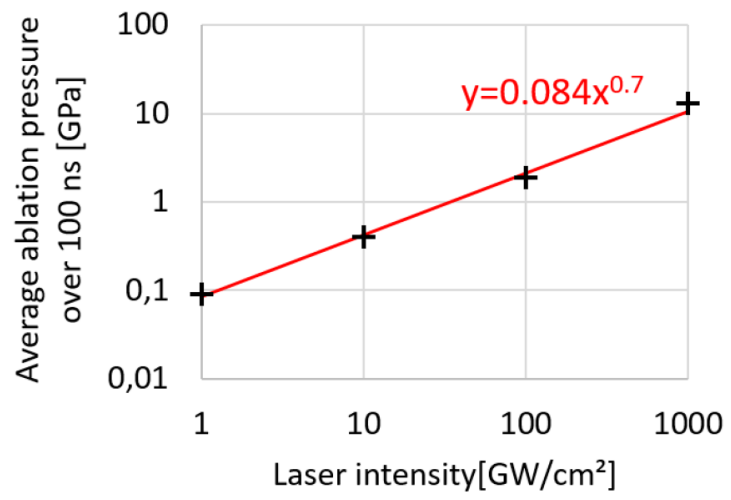

FIG. 6. Pressure/intensity law obtained with the ESTHER code in the case of square pulses of $100 \mathrm{~ns}$. The pressure has been averaged over the pulse duration.

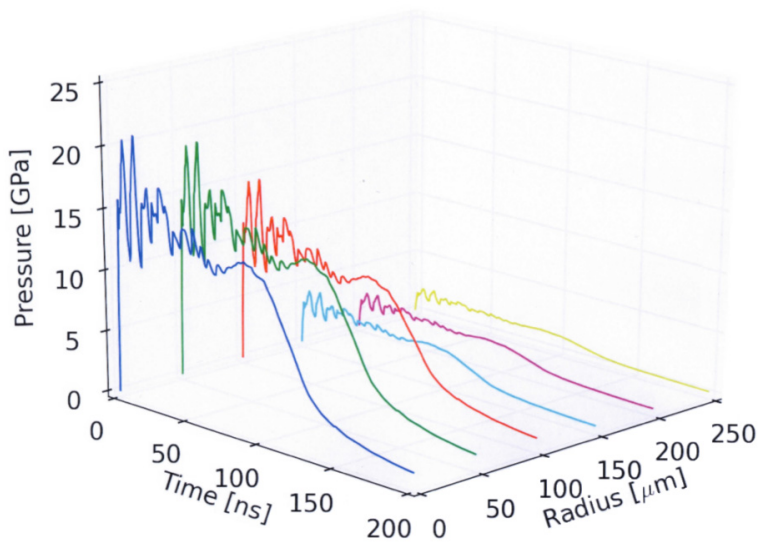

FIG. 7. 2D-axisymmetrical ablation pressure law $P_{\mathrm{ab}}(r, t)$ for a $39.5 \mathrm{~J}$ shot with a $100 \mathrm{~ns}$ pulse duration and a $220 \mu \mathrm{m}$ focal spot diameter on an aluminum target.

the following equations:

$$
\begin{aligned}
G_{\mathrm{SCG}}= & G_{0}\left[1+\frac{G_{p}}{G_{0}}\left(\frac{\rho_{0}}{\rho}\right)^{1 / 3} P+\frac{G_{T}}{G_{0}}(T-300)\right], \\
Y_{\mathrm{SCG}}= & \min \left\{Y_{0}\left[1+\beta\left(\epsilon+\epsilon_{i}\right)\right]^{n} ; Y_{\max }\right\} \\
& \times\left[1+\frac{G_{p}}{G_{0}}\left(\frac{\rho_{0}}{\rho}\right)^{1 / 3} P+\frac{G_{T}}{G_{0}}(T-300)\right] .
\end{aligned}
$$

The parameters $Y_{0}, Y_{\max }, G_{0}, G_{p}, G_{T}, \rho_{0}, \beta, n$, and $\epsilon_{i}$ are defined elsewhere. ${ }^{28}$ The present targets have been extracted from a massive piece of 6061-T6 aluminum which has been used in the past for various experiments. ${ }^{29-31}$ In our simulation, we use the parameters of the SCG model which have been calibrated and validated on these experiments. They are presented in Table I.

The numerical results obtained using the HESIONE code are compared to experimental measurements of free surface velocities in Fig. 8. For these Eulerian simulations, a Cartesian mesh was used with a mesh size of $2 \mu \mathrm{m} \times 2 \mu \mathrm{m}$. Globally, the four velocities are well reproduced by our simulation. Specifically, there is good agreement on the second arrival of the shock at $90 \mathrm{~ns}$.

\section{Discussion}

This good agreement validates our modeling of laser loading. Specifically, the use of an average fluence on the central plateau of the focal spot and our extrapolation of the radial profile $g(r)$ (see Fig. 3).

Spatial heterogeneities present in the focal spot generate transverse mechanical waves inside the target which lead to a smoothing of the stress wave traveling through the sample thickness. We can affirm that a thickness of $188 \mu \mathrm{m}$ is sufficient to completely smooth these heterogeneities.

It is interesting to note that the laser intensity decreases by two orders of magnitude in $750 \mu \mathrm{m}$ according to our exponential extrapolation, whereas the velocity of the rear surface only decreases by one order of magnitude in $750 \mu \mathrm{m}$. Such a difference is attributed to 
TABLE I. Set of parameters used for the SCG model of 6061-T6 aluminum.

\begin{tabular}{lccccccc}
\hline \hline$Y_{0}(\mathrm{GPa})$ & $Y_{\max }(\mathrm{GPa})$ & $G_{0}(\mathrm{GPa})$ & $G_{p}$ & $G_{T}\left(\mathrm{GPa} \mathrm{K}^{-1}\right)$ & $\rho_{0}\left(\mathrm{~kg} \mathrm{~m}^{-3}\right)$ & $\beta$ & $n$ \\
\hline 0.4 & 0.5 & 27.6 & 1.79952 & -0.0170016 & 2700 & 5550 & 0.1 \\
\hline \hline
\end{tabular}

edge effects that are predominant in our experimental configuration. Indeed, release waves are generated at the focal spot edges. When these waves reach the center of the spot, they reduce the shock level in this location. Simultaneously, stress waves are generated and
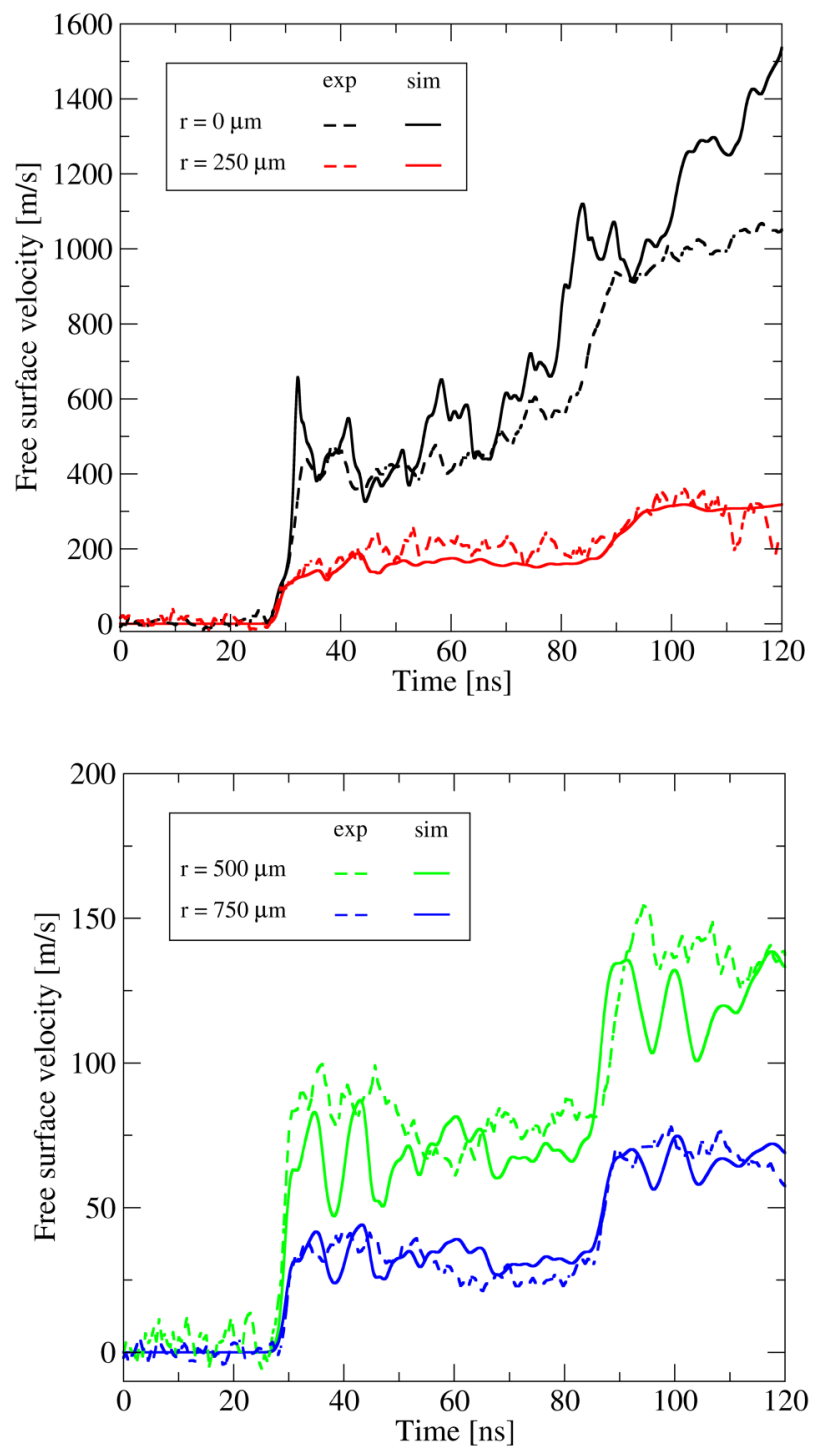

FIG. 8. Comparison between experimental (dotted lines) and numerical results (solid lines) for free surface velocities of a thin aluminum target at different radii. Note the different scales for the two graphs. propagate outwards, increasing the stress (and the rear surface velocity) at locations away from the center of the focal spot.

In conclusion, good agreement observed between numerical and experimental results valids our methodology of simulation. Now, we can use it to simulate cratering experiments on thick targets.

\section{APPLICATION TO LASER-DRIVEN CRATERING}

\section{A. Experimental results}

Seven shots were performed on the GCLT facility at energies between 3 and $36 \mathrm{~J}$ on $3 \mathrm{~mm}$ thick 6061-T6 aluminum targets. This thickness being very large compared to dimensions of resulting craters, targets can be considered as semi-infinite. The complete experimental setup is described in Sec. IV.

All the craters have been measured using an interferometric profilometer. It provides us a 3D map of each crater from which we extract a profile crossing the crater center. At high energy $(>20 \mathrm{~J})$, craters can be considered as axisymmetrical, so the extracted profile does not depend of the direction chosen for the cut. The profile of the crater obtained with an energy of $20.6 \mathrm{~J}$ is shown in Fig. 9. Contrariwise, at low energy $(<20 \mathrm{~J})$, there are some hollows at the bottom of the craters, so they are not perfectly axisymmetrical. These hollows are probably the consequence of the inhomogeneities observed in the focal spot [see Fig. 2(b)].

On each crater profile, we measure the maximum depth $d$, the diameter $\phi$ and the volume $V$. We define these quantities relative to the initial surface of the sample. It is possible to precisely measure $d$, but there are some uncertainties for $\phi$ and $V$. Indeed, there are no lips around the craters so the intersection with the initial surface is not very precise. To bypass this problem, we fit each crater profile by a parabola of the following form:

$$
p(r)=a r^{2}-d
$$

where $r$ is the radial coordinate and $a$ is a real. Then, $\phi$ and $V$ are

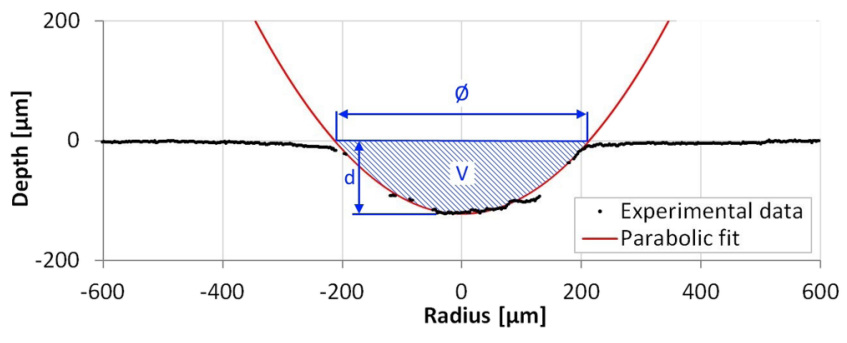

FIG. 9. $2 \mathrm{D}$ profile of the crater generated by the $20.6 \mathrm{~J}$ shot. This profile is fitted by a parabola to allows the definition of $d, \phi$ and $V$. 
TABLE II. Summary of experimental measurements for craterization laser shots on thick aluminum targets.

\begin{tabular}{lccc}
\hline \hline$E(\mathrm{~J})$ & $d(\mu \mathrm{m})$ & $\phi \pm 10 \%(\mu \mathrm{m})$ & $V \pm 20 \%\left(\times 10^{6} \mu \mathrm{m}^{3}\right)$ \\
\hline 3.7 & 12 & $\ldots$ & $\ldots$ \\
7.0 & 27 & $\ldots$ & $\ldots$ \\
10.1 & 44 & 368 & 2.3 \\
15.8 & 95 & 406 & 6.2 \\
20.6 & 122 & 425 & 8.7 \\
28.2 & 155 & 455 & 12.6 \\
36.0 & 204 & 513 & 21.1 \\
\hline \hline
\end{tabular}

defined as follows:

$$
\phi=2 \sqrt{\frac{d}{a}} \text { and } \quad V=\left|\int_{0}^{\phi / 2} 2 \pi r p(r) d r\right|=\frac{\pi}{8} d \phi^{2} .
$$

The overall dimensions are summarized in Table II. The shapes of the two smallest craters cannot be fitted by parabolas, so we do not measure $\phi$ and $V$ for these two craters.

We consider that the uncertainty about $d$ is negligible and the uncertainty about $\phi$ is close to $10 \%$, so, according to Eqs. (18) and (19), the uncertainty about $V$ is $20 \%$. These uncertainties are mainly due to the fact that craters are not perfectly axisymmetric and their shapes are not exactly parabolic.

\section{B. Numerical simulations}

Figure 10 shows a comparison between experimental and numerical profiles for the $20.6 \mathrm{~J}$ shot. The simulation was stopped $1.5 \mu$ s after the laser deposit because the quantities $d, \phi$, and $V$ no longer evolve afterwards. The experimental crater depth is well reproduced, but the shape of the crater edges is different. Our simulation predicts the formation of lips around the crater, whereas these lips are not observed experimentally. Therefore, the diameter is underestimated by simulation.

The seven experimental shots presented above have been simulated. From each simulation, the crater profile has been extracted and the quantities $d, \phi$, and $V$ have been measured. A comparison of these quantities with experimental data of Table II is shown in Fig. 11. Concerning $d$, simulated data underestimate

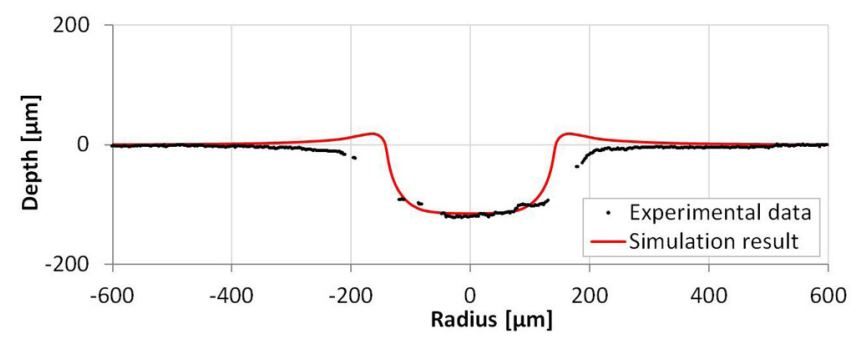

FIG. 10. Comparison between experimental and numerical crater profiles for the $20.6 \mathrm{~J}$ shot (simulation stopped $1.5 \mu$ s after the laser shot). the measurements at low energy $(<20 \mathrm{~J})$, but a good agreement is observed at high energy $(>20 \mathrm{~J})$. Given that $d$ is defined as the maximal depth, the presence of hollows at the bottom of experimental craters at low energy can explain this difference. $\phi$ is always underestimated by the simulation because the crater edges are never well calculated as for the $20.6 \mathrm{~J}$ shot presented in

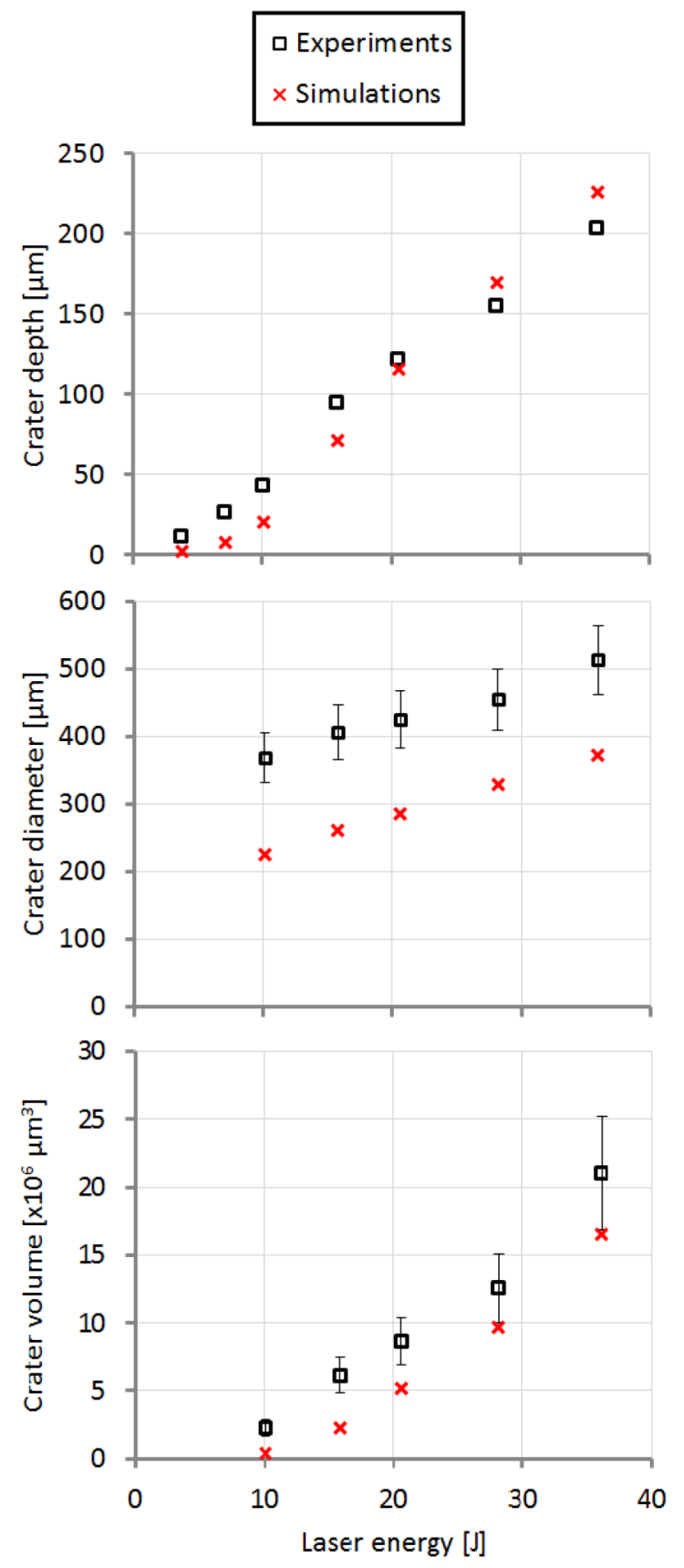

FIG. 11. Evolution of $d, \phi$, and $V$ as a function of the laser energy. 


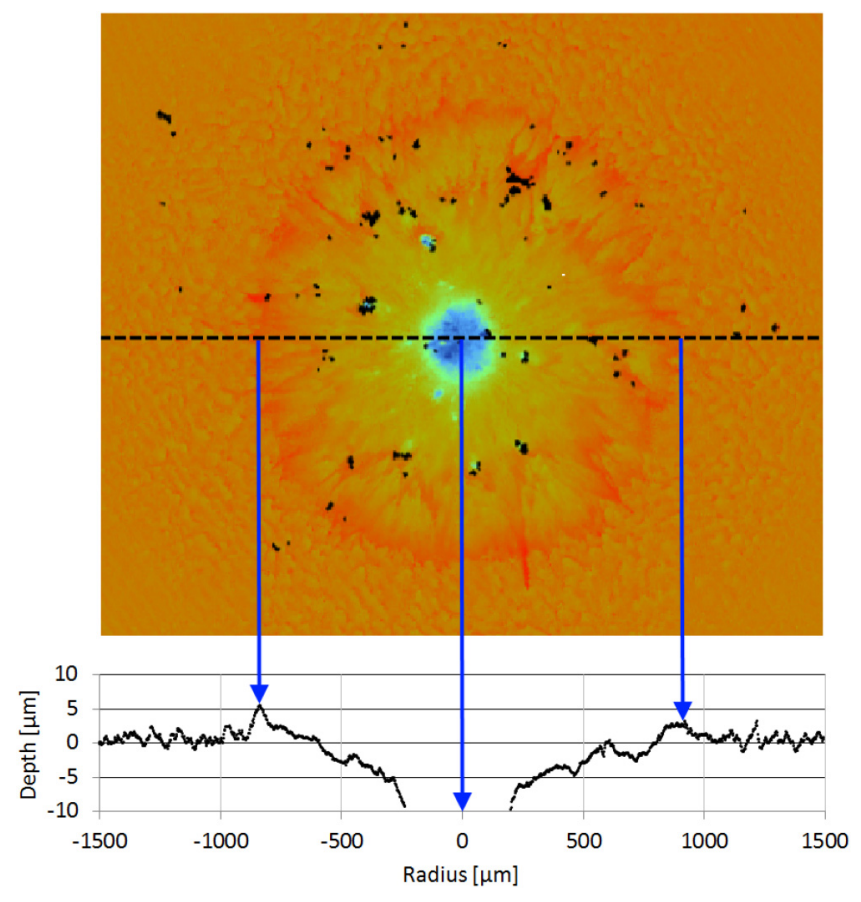

FIG. 12. Top view of the $20.6 \mathrm{~J}$ crater (top) and the corresponding $2 \mathrm{D}$ profile according to the cutting plane represented in a dotted line (bottom). Both have been obtained using an optical profilometer. Note that the black profile shown here is identical to the black profile shown in Fig. 10, but scales are very different between the two figures.

Fig. 10. However, the trend followed by $\phi$ is well predicted by the simulation. Logically, $V$ is also underestimated. We notice that the relative error decreases as the energy increases.

\section{Discussion}

Some of the differences that have been pointed out could be due to thermal effects which are visible all around the crater as shown in Fig. 12. On this top view of the $20.6 \mathrm{~J}$ crater, we observe that melted matter has been ejected during crater formation. The same projections have been observed in Ref. 32. In order to better appreciate this phenomenon, the $2 \mathrm{D}$ profile is plotted under the top view with the same radial scale. This profile is identical to the black profile shown in Fig. 10, but scales are very different between the two figures. The effect of a laser pressure applied on a liquid surface is studied in Ref. 33. The ejected matter is materialized by a small extra thickness of a few micrometers deposited all around the crater at a distance of about $1 \mathrm{~mm}$ from the center. More precisely, we suppose that the interaction of the laser with the matter melts a thin layer of aluminum and mechanical effects project it all around the crater. During its ejection, this melted matter can erode solid material on the edges of the crater. This could explain the fact that experimental craters do not have lips.

Our simulation method cannot reproduce this phenomenon because the laser/matter interaction and the mechanical response of the material are treated with two different codes. In the HESIONE simulations, the pressure is applied to a target which is initially cold and solid. Moreover, the thermal conductivity is neglected in these simulations.

For the shots presented in this paper, the thickness of matter affected by thermal effects varies between 10 and $30 \mu \mathrm{m}$ depending on the laser intensity. This thickness is small compared to the crater depth at high intensities, but it is of the same order of magnitude at low intensities. In the latter case, thermal effects cannot be neglected in view of the mechanical effects. It explains the large difference observed on the crater depth at low energies in Fig. 11. Moreover, at low energies, the inhomogeneities in the focal spot generate inhomogeneities in the stress wave. Our 2D-axisymmetrical simulations are not able to take this phenomenon into account.

\section{CONCLUSION}

A methodology to simulate craterization laser shots have been proposed. A first code is used to treat the laser/matter interaction and determine the pressure generated by the laser on the target surface. This pressure is then used as a boundary condition of a second code to simulate the shock propagation and the mechanical response of the material. This second code is Eulerian, so simulations are robust and well adapted to the large deformations observed during the cratering process.

The methodology has been applied to simulate a series of nanosecond laser shots on semi-infinite targets of 6061-T6 aluminum. Comparing experimental and numerical crater profiles, some differences are observed. For the most part, they can be explained by thermal effects, which can be significant in this laser regime. However, our simulations are able to predict the evolution of craters' dimensions as a function of the laser energy.

The authors are interested in the effect of laser irradiation on other materials such as brittle materials. Experimental results on a porous graphite have already been presented in Ref. 8. Numerical simulations of these laser shots will be simulated following the present methodology which remains valid for this type of material.

\section{REFERENCES}

${ }^{1}$ B. G. Cour-Palais, “A career in applied physics: Apollo through space station," Int. J. Impact Eng. 23, 137-168 (1999).

${ }^{\mathbf{2}}$ W. Harrison, C. Loupias, P. Outrebon, and D. Turland, "Experimental data and hydrocode calculations for hypervelocity impacts of stainless steel into aluminium in the 2-8 km/s range," Int. J. Impact Eng. 17, 363-374 (1995).

${ }^{3}$ S. Latunde-Dada, C. Cheesman, D. Day, W. Harrison, and S. Price, "Hypervelocity impacts into graphite," J. Phys.: Conf. Ser. 63286, 012042 (2011).

${ }^{4}$ P. E. Nebolsine, in Proceedings of the AIAA 14th Aerospace Sciences Meeting, Washington, DC, 26-28 January 1976 (AIAA, Washington, DC, 1976).

${ }^{5}$ A. N. Pirri, "Theory for laser simulation of hypervelocity impact," Phys. Fluids 20, 221-228 (1977).

${ }^{6}$ A. Deom, D. De Lavergne, and D. L. Balageas, in Proceedings of the 6th International Conference on Erosion by Liquid and Solid Impact, Cambridge, UK, 5-8 September 1983, (Cavendish Laboratory, Cambridge UK, 1983).

${ }^{7}$ G. Seisson, D. Hebert, I. Bertron, J.-M. Chevalier, L. Hallo, E. Lescoute, L. Videau, P. Combis, F. Guillet, M. Boustie, and L. Berthe, "Dynamic cratering of graphite: Experimental results and simulations," Int. J. Impact. Eng. 63, 18-28 (2014). 
${ }^{8}$ B. Aubert, D. Hebert, J.-L. Rullier, I. Bertron, F. Malaise, L. Videau, E. Lescoute, and L. Berthe, in Proceedings of the 22nd DYMAT Technical Meeting, Grenoble, France, 19-21 October 2016 (EDP Sciences, Web of Conferences, 2016).

${ }^{9}$ L. Berthe, R. Fabbro, P. Peyre, L. Tollier, and E. Bartnicki, "Shock waves from a water-confined laser-generated plasma," J. Appl. Phys. 82, 2826 (1997).

${ }^{10} \mathrm{M}$. Boustie and F. Cottet, "Experimental and numerical study of laser induced spallation into aluminum and copper targets," J. Appl. Phys. 69, 7533 (1991).

${ }^{11}$ F. Z. Dai, J. Z. Lu, Y. K. Zhang, D. P. Wen, X. D. Ren, and J. Z. Zhou, "Effect of laser spot size on the residual stress field of pure al treated by laser shock processing: Simulations," Appl. Surf. Sci. 316, 477-483 (2014).

${ }^{12}$ M. Boustie, J.-P. Cuq-Lelandais, C. Bolis, L. Berthe, S. Barradas, M. Arrigoni, T. De Resseguier, and M. Jeandin, "Study of damage phenomena induced by edge effects into materials under laser driven shocks," J. Phys. D: Appl. Phys. 40, 7103-7108 (2007).

${ }^{13}$ T. De Resseguier, P. Berterretche, and M. Hallouin, "Influence of quartz anisotropy on shock propagation and spall damage," Int. J. Impact Eng. 31, 545-557 (2005)

${ }^{14}$ C. S. Debonnel, J. L. Vierne, F. Bonneau, and P. Combis, in Proceedings of the International Topical Meeting on Mathematics and Computation and Supercomputing in Nuclear Applications, Monterey, CA, 15-19 April 2007 (American Nuclear Society, La Grange Park, IL, 2007).

${ }^{15} \mathrm{~L}$. M. Barker and R. E. Hollenbach, "Laser interferometer for measuring high velocities of any reflecting surface," J. Appl. Phys. 43, 4669-4675 (1972).

${ }^{16} \mathrm{~W}$. Herrmann and J. S. Wilbeck, "Review of hypervelocity penetration theories," Int. J. Impact Eng. 5, 307-322 (1987).

17J. Grun, R. Decoste, B. H. Rippin, and J. Gardner, "Characteristics of ablation plasma from planar, laser-driven targets,” Appl. Phys. Lett. 39, 545-547 (1981).

${ }^{18}$ C. R. Phipps, T. P. Turner, R. F. Harrison, G. W. York, W. Z. Osborne, G. K. Anderson, X. F. Corlis, L. C. Haynes, H. S. Steele, and T. R. Spicochi, "Impulse coupling to targets in vacuum by $\mathrm{KrF}, \mathrm{HF}$, and $\mathrm{CO}_{2}$ single-pulse lasers," J. Appl. Phys. 64, 1083 (1988).

${ }^{19}$ R. Dautray and J.-P. Watteau, La Fusion Thermonucleaire par Laser (CEA, Paris, 1993).

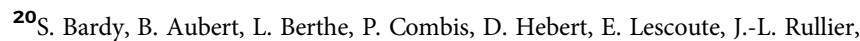
and L. Videau, "Numerical study of laser ablation on aluminum for shock-wave applications: Development of a suitable model by comparison with recent experiments," Opt. Eng. SPIE 56, 011014 (2017)

${ }^{21}$ P. Combis and L. Videau, "Notice d'utilisation du code esther version 4," CEA Internal Technical Report (CEA, Paris, 2010).

${ }^{22}$ G. Seisson, D. Hebert, I. Bertron, L. Videau, P. Combis, L. Berthe, and M. Boustie, in Proceedings of the 18th APS-SCCM, Bristol, UK, 2013 (Institute of Physics, 2014)

${ }^{23}$ P. Woodward and P. Collela, "The numerical simulation of two-dimensional fluid flow with strong shocks,” J. Comput. Phys. 54, 115-173 (1984).

${ }^{24}$ Y. S. Touloukian, P. E. Liley, and S. C. Saxena, Thermophysical Properties of Matter, Thermal Conductivity, Vol. 1 (IFI Plenum, New York, 1970).

${ }^{25}$ D. Palik, Handbook of Optical Constants of Solids (Academic, New York, 1985).

${ }^{26}$ W. Ebeling, A. Forster, V. E. Fortov, V. K. Gryaznov, and A. Y. Polishchuk, Thermophysical Properties of Hot Dense Plasmas (TeubnerTexte, Stuttgart, 1991).

${ }^{27}$ A. Decoster, P. A. Markowich, and B. Perthame, Modeling of Collisions (Gauthier-Villars, Paris, 1998).

${ }^{28}$ D. J. Steinberg, S. G. Cochran, and M. W. Guinan, "A constitutive model for metals applicable at high strain rate," J. Appl. Phys. 51, 1498-1504 (1980).

${ }^{29}$ P. Pradel, F. Malaise, B. Cadilhon, T. de Resseguier, and C. Delhomme, in Proceedings of the 1st ICILSM, Turin, Italy, May 2016 (Politecnico di Torino, Turin, 2016).

${ }^{30} \mathrm{~B}$. Jodar, "Damage and fragmentation of a porous graphite under high dynamic pressures," M.Sc. thesis, Technische Universitat Braunschweig, 2014.

${ }^{31}$ D. Hebert, L. Hallo, L. Voisin, T. Desanlis, A. Galtie, B. Bicrel, C. Maunier, P. Mercier, and G. Duchateau, "A kdp equation of state for laser induced damage applications," J. Appl. Phys. 109, 123527 (2011).

${ }^{32}$ B. Arad, S. Eliezer, I. Gilath, E. Moshe, and C. G. Simon, "Laser simulation of hypervelocity impacts in space," Trans. Built Environ. 19, 431-440 (1996).

${ }^{33} \mathrm{~V}$. V. Semak and A. Matsunawa, "The role of recoil pressure in energy balance during laser material processing,” J. Phys. D: Appl. Phys. 30, 2541-2552 (1997). 